\title{
TomTom for Business Process Management (TomTom4BPM)
}

\author{
Wil M.P. van der Aalst \\ Eindhoven University of Technology \\ P.O. Box 513, NL-5600 MB, Eindhoven, The Netherlands \\ w.m.p.v.d.aalst@tue.nl
}

\begin{abstract}
Navigation systems have proven to be quite useful for many drivers. People increasingly rely on the devices of TomTom and other vendors and find it useful to get directions to go from $\mathrm{A}$ to $\mathrm{B}$, know the expected arrival time, learn about traffic jams on the planned route, and be able to view maps that can be customized in various ways (zoomin/zoom-out, show fuel stations, speed limits, etc.). However, when looking at business processes, such information is typically lacking. Good and accurate "maps" of business process are often missing and, if they exist, they tend to be restrictive and provide little information. For example, very few business process management systems are able to predict when a case will complete. Therefore, we advocate more TomTom-like functionality for business process management (TomTom4BPM). Process mining will play an essential role in providing TomTom4BPM as it allows for process discovery (generating accurate maps), conformance checking (comparing the real processes with the modeled processes), and extension (augmenting process models with additional/dynamic information).
\end{abstract}

\section{The Need for Process Navigation}

Business Process Management Systems (BPMSs) [15/8] are used to manage and execute operational processes involving people, applications, and/or information sources on the basis of process models. These systems can be seen as the next generation of workflow technology offering more support for analysis. Despite significant advances in the last decade, the functionality of today's BPMSs leaves much to be desired. This becomes evident when comparing such systems with the latest car navigation systems of TomTom that provide detailed maps, real-time traffic information, re-routing, customized points of interest, estimated arrival times, etc. (cf. Figure 1). Some examples of TomTom-like functionality that is generally missing are listed below:

- In today's organizations often a good process map is missing. Process models are not present, incorrect, or outdated. Sometimes process models are used to directly configure the BPMS. However, in most situations there is not an explicit process model as the process is fragmented and hidden inside legacy code, the configuration of ERP systems, and in the minds of people. 


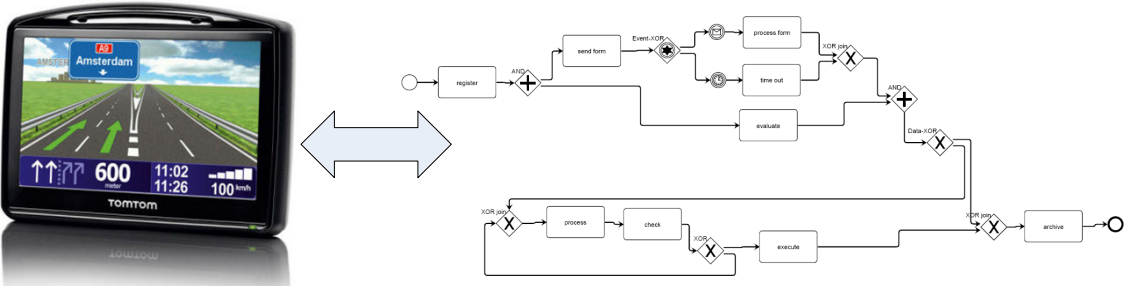

Fig. 1. Comparing maps in a navigation system with maps in a BPMS

- If process models exist in an explicit form, their quality typically leaves much to be desired. Especially when a process model is not used for enactment and is only used for documentation and communication, it tends to present a "PowerPoint reality". Road maps are typically of much higher quality and use intuitive colors and shapes of varying sizes, e.g., highways are emphasized by thick colorful lines and dirt roads are not shown or shown using thin dark lines. In process models, all activities tend to have the same size and color and it is difficult to distinguish the main process flow from the less traveled process paths.

- Most process modeling languages have a static decomposition mechanism (e.g., nested subprocesses). However, what is needed are controls allowing users to zoom in or zoom out seamlessly like in a navigation system or Google maps. Note that, while zooming out, insignificant things are either left out or dynamically clustered into aggregate shapes (e.g., streets and suburbs amalgamate into cities). Process models should not be static but allow for various views.

- Sometimes process models are used for enactment. However, such "process maps" are controlling the users. When using a car navigation system, the driver is always in control, i.e., the road map (or TomTom) is not trying to "control" the user. The goal of a BPMS should be to provide directions and guidance rather than enforcing a particular route.

- A navigation system continuously shows a clear overview of the current situation (i.e., location and speed). Moreover, traffic information is given, showing potential problems and delays. This information is typically missing in a BPMS. Even if the BPMS provides a management dashboard, TomTom-like features such as traffic information and current location are typically not shown in an intuitive manner.

- A TomTom system continuously recalculates the route, i.e., the recommended route is not fixed and changed based on the actions of the driver and contextual information (e.g. traffic jams). Moreover, at any point in time the navigation system is showing the estimated arrival time. Existing BPMSs are not showing this information and do not recalculate the optimal process based on new information. 
The above list of examples illustrates desirable functionality that is currently missing in commercial BPMSs. Fortunately, recent breakthroughs in process mining may assist in realizing TomTom-like functionality for business process management (TomTom4BPM).

\section{Process Mining}

Process mining techniques attempt to extract non-trivial and useful information from event logs [2]. Many of today's information systems are recording an abundance of events in such logs. Various process mining approaches make it possible to uncover information about the processes they support. Typically, these approaches assume that it is possible to sequentially record events such that each event refers to an activity (i.e., a well-defined step in the process) and is related to a particular case (i.e., a process instance). Furthermore, some mining techniques use additional information such as the performer or originator of the event (i.e., the person/resource executing or initiating the activity), the timestamp of the event, or data elements recorded with the event (e.g., the size of an order).

Process mining addresses the problem that most people have very limited information about what is actually happening in their organization. In practice, there is often a significant gap between what is prescribed or supposed to happen, and what actually happens. Only a concise assessment of the organizational reality, which process mining strives to deliver, can help in verifying process models, and ultimately be used in a process redesign effort or BPMS implementation.

Some examples of questions addressed by process mining:

- Process discovery: "What is really happening?"

- Conformance checking: "Do we do what was agreed upon?"

- Performance analysis: "Where are the bottlenecks?"

- Process prediction: "Will this case be late?"

- Process improvement: "How to redesign this process?"

These examples show that process mining is an important enabler for TomTom4BPM, i.e., TomTom-like functionality for business processes. This can be demonstrated by looking at the functionality of ProM [2].

- ProM's Fuzzy Miner [6] can discover processes from event logs and offers a seamless zoom similar to TomTom or Google Maps.

- ProM's Recommendation Engine 7] learns from historic data and uses this to provide recommendations to the user. This way the workflow system can provide more flexibility while still supporting the user. This is comparable to the directions given by a navigation system.

- ProM's Prediction Engine 44 also learns for historic data but now uses this information to make predictions, e.g., the estimated completion time of a case or the likelihood the occurrence of a particular activity.

The interested reader is referred to www. processmining. org for more information about these ideas and for downloading the ProM software. 


\section{References}

1. van der Aalst, W.M.P.: Business Process Management Demystified: A Tutorial on Models, Systems and Standards for Workflow Management. In: Desel, J., Reisig, W., Rozenberg, G. (eds.) Lectures on Concurrency and Petri Nets. LNCS, vol. 3098, pp. 1-65. Springer, Heidelberg (2004)

2. van der Aalst, W.M.P., Reijers, H.A., Weijters, A.J.M.M., van Dongen, B.F., Alves de Medeiros, A.K., Song, M., Verbeek, H.M.W.: Business Process Mining: An Industrial Application. Information Systems 32(5), 713-732 (2007)

3. van der Aalst, W.M.P., Weijters, A.J.M.M., Maruster, L.: Workflow Mining: Discovering Process Models from Event Logs. IEEE Transactions on Knowledge and Data Engineering 16(9), 1128-1142 (2004)

4. van Dongen, B.F., Crooy, R.A., van der Aalst, W.M.P.: Cycle Time Prediction: When Will This Case Finally Be Finished. In: Meersman, R., Tari, Z. (eds.) CoopIS 2008, OTM 2008, Part I. LNCS, vol. 5331, pp. 319-336. Springer, Heidelberg (2008)

5. Dumas, M., van der Aalst, W.M.P., ter Hofstede, A.H.M.: Process-Aware Information Systems: Bridging People and Software through Process Technology. Wiley \& Sons, Chichester (2005)

6. Günther, C.W., van der Aalst, W.M.P.: Fuzzy Mining: Adaptive Process Simplification Based on Multi-perspective Metrics. In: Alonso, G., Dadam, P., Rosemann, M. (eds.) BPM 2007. LNCS, vol. 4714, pp. 328-343. Springer, Heidelberg (2007)

7. Schonenberg, H., Weber, B., van Dongen, B.F., van der Aalst, W.M.P.: Supporting Flexible Processes Through Recommendations Based on History. In: Dumas, M., Reichert, M., Shan, M.-C. (eds.) BPM 2008. LNCS, vol. 5240, pp. 51-66. Springer, Heidelberg (2008)

8. Weske, M.: Business Process Management: Concepts, Languages, Architectures. Springer, Berlin (2007) 